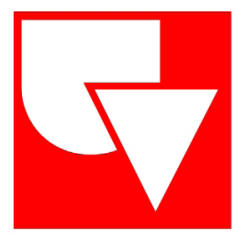

Universidad

del Valle

\title{
REVISTA ESTOMATOLOGIA
}

Escuela de Odontología Facultad de Salud

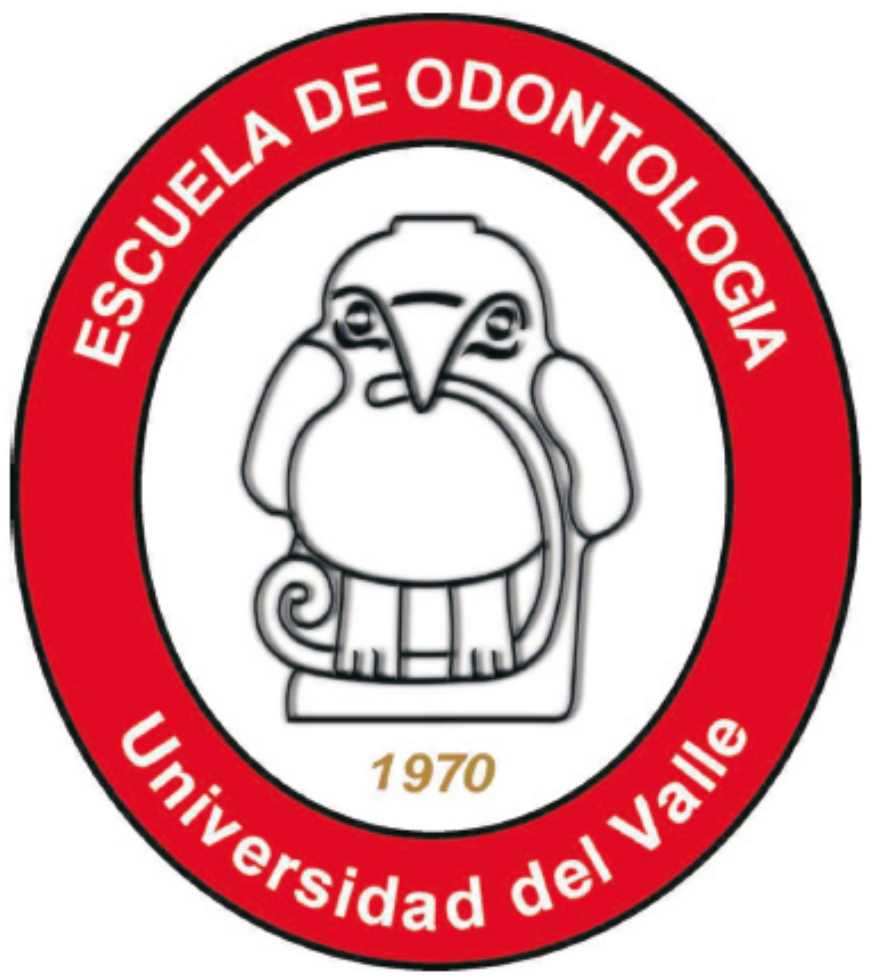

CONTENIDO

\begin{tabular}{|c|c|}
\hline 5 & $\begin{array}{l}\text { EDITORIAL } \\
\text { ANDRÉS FELIPE AGUIRRE }\end{array}$ \\
\hline 6 & $\begin{array}{l}\text { ARTÍCULO DE OPINIÓN } \\
\text { EL RECURSO HUMANO EN SALUD ORAL: ODONTÓLOGOS Y AUXILIARES EN SALUD ORAL EN BOGOTÁ D. C., } \\
\text { COLOMBIA } \\
\text { JAIRO H. TERNERA }\end{array}$ \\
\hline 8 & $\begin{array}{l}\text { ARTÍCULOS ORIGINALES } \\
\text { ESTADO BUCODENTAL EN GESTANTES DE LA CIUDAD DE ARMENIA, COLOMBIA } \\
\text { HERNEY A. RENGIFO, FRANCY CIRO, ALEJANDRA OBANDO, LINA ROLDAN, ANDREA LOPEZ, LINA LOPEZ }\end{array}$ \\
\hline 13 & $\begin{array}{l}\text { PREVALENCIA DE CARIES Y ALTERACIONES DENTARIAS EN NIÑOS CON LABIO FISURADO Y PALADAR } \\
\text { HENDIDO DE UNA FUNDACIÓN DE SANTIAGO DE CALI } \\
\text { ANA M. HURTADO, LINA M. ROJAS, DIANA M. SANCHEZ, ANA M. GARCÍA, MARIAA. ORTÍZ, ANDRÉS F. AGUIRRE }\end{array}$ \\
\hline 18 & $\begin{array}{l}\text { EFECTOS DEL PERÓXIDO DE HIDRÓGENO ACTIVADO CON LUZ ULTRAVIOLETA Y EL PERÓXIDO DE CARBAMI- } \\
\text { DA EN ACLARAMIENTO DENTAL } \\
\text { ADRIANA CABRERA, MELISSA DAVID, LUISA S. PACHECO, ANA C. SUÇAREZ, HERNEY GARZÓN }\end{array}$ \\
\hline 25 & $\begin{array}{l}\text { REVISIÓNES DE TEMA } \\
\text { REABSORCIÓN RADICULAR INDUCIDA POR ORTODONCIA } \\
\text { ANGIE CLARKSON }\end{array}$ \\
\hline 30 & $\begin{array}{l}\text { DIFERENCIAS ENTRE EL NIVEL DE SIGNIFICANCIA ALFA Y EL VALOR P } \\
\text { HECTOR F. MUESES. }\end{array}$ \\
\hline
\end{tabular}

Cali - Valle del Cauca, Colombia 


\section{DIRECTIVAS}

Doctor IVÁN ENRIQUE RAMOS

Rector de la Universidad

Doctora MARTHA CECILIA GÓMEZ DE GARCÍA

Vicerrectora Académica

Doctor JOSÉ FRANCISCO PARRA

Vicerrector Administrativo

Doctora CAROLINA ISAZA

Vicerrectora de Investigaciones

Doctora LIBIA SOTO

Vicerrectora de Bienestar Universitario

Doctora LILIANA ARIAS

Decana Facultad de Salud

Doctor JESÚS ALBERTO HERNÁNDEZ

Director Escuela de Odontología

Doctora JUDY ELENA VILLAVICENCIO

Directora Programa Académico

Doctor JORGE ENRIQUE SOTO

Director de Postgrados

Doctor JESÚS ALBERTO CALERO

Director Programa de Auxiliar de Salud Oral

DIRECTOR EDITOR: ADOLFO CONTRERAS EDITOR ASOCIADO: JOSÉ FERNANDO BARRETO

CONSEJO EDITORIAL DE HONOR

\author{
GERARDO UMAÑA \\ BEATRIZ GÓMEZ DE ARISMENDI \\ JOSÉ DOMINGO GARCÍA \\ JESÚS ALBERTO HERNÁNDEZ \\ LIBIA SOTO \\ DIEGO VALLEJO
}

\section{CONSEJO EDITORIAL}

Escuela de Odontología

ADOLFO CONTRERAS PhD.

JOSÉ FERNANDO BARRETO MSC.

ADRIANA JARAMILLO MSC.

ANDRÉS FELIPE AGUIRRE

CARLOS MARTÍNEZ, EDITOR ON LINE

FREDDY MORENO, EDITOR ASOCIADO ON LINE

YOLANDA CHAPARRO DE VICTORIA

IMPRENTA DEPARTAMENTAL DEL VALLE DEL CAUCA

Edificio de la Gobernación del Valle 8855253 - 8895926

ISSN 0121-3873

\section{COMITÉ CIENTÍFICO NACIONAL}

Alejandro Botero Ph.D., Fac Odontología Universidad de Antioquia

Javier Botero Ph.D., Fac Odontología Universidad de Antioquia

Beatriz Parra Ph.D., Esc Ciencias Básicas Universidad del Valle

Gloria Inés Lafaurie, Fac Odontología Universidad el Bosque

Carlos Corredor Ph.D., Universidad Javeriana

\section{COMITÉ CIENTÍFICO INTERNACIONAL}

Rodolfo Acosta MSc., Nova Soutwest University, EE.UU

Rubén León Ph.D., Universidad De Chile,

Jorge Gamonal Ph.D., Universidad De Chile, Santiago

Roger Arce MSc, University of North Carolina, EE.UU

Giussepe Merlati, Departamento de Odontoestomatologia, Universidad de Pavia, Italia.

Claudia De León, Editora Revista Mexicana de Odontología, Unam, México D.F.

\author{
http://odontologia.univalle.edu.co \\ e-mail: odontologia@univalle.edu.co
}

La Revista Estomatología, órgano oficial de la Escuela de Odontología de la Universidad del Valle, aparece semestralmente. Publica artículos científicos originales inéditos sobre odontoestomatología, ciencias afines de la salud, ciencias naturales, sociales y humanas, incluidos los de tipo cultural y tecnológico, así como la programación general y la información académica de la Escuela y de la Facultad de Salud de la Universidad del Valle.

Los artículos publicados en la Revista Estomatología son de la responsabilidad exclusiva del autor o de los autores y no necesariamente reflejan el pensamiento del editor o del Comité Editorial.

El contenido de esta publicación puede ser citado o copiado, siempre y cuando se haga referencia adecuada al autor o a los autores de los artículos que se incluyen en la Revista.

Las cartas al Editor se deben dirigir a:

Dr. Adolfo Contreras

Revista Estomatología

Escuela de Odontología, Facultad de Salud, Universidad del Valle.

San Fernando, Calle 3 No. 36B-00 Edificio 132

Apartado Aéreo 25360, Cali, Colombia
Manuscritos y otra correspondencia a:

Revista Estomatología odontologia@univalle.edu.co

Dr. José Fernando Barreto, Editor Asociado Escuela de Odontología, Facultad de Salud

Universidad del Valle, San Fernando Calle 3a N³6B-00, Edificio 132 Oficina 307 Apartado Aéreo 25360, Cali, Colombia 


\title{
REVISTA ESTOMATOLOGÍA
}

\author{
ESCUELA DE ODONTOLOGÍA \\ FACULTAD DE SALUD, UNIVERSIDAD DEL VALLE \\ Volumen $16 \mathrm{~N}^{\circ} 12008$ \\ Contenido
}

5 EDITORIAL

Andrés Felipe Aguirre

Artículo de Opinión

6 EL RECURSO HUMANO EN SALUD ORAL: ODONTÓLOGOS Y AUXILIARES EN SALUD ORAL EN BOGOTA, COLOMBIA

THE HUMAN SOURCE IN ORAL HEALTH: DENTIST AND ORAL HEALTH ASSISTANT IN BOGOTÁ CITY, COLOMBIA

JaIRo H. TERnERA

Articulos Originales

8 ESTADO BUCODENTAL EN GESTANTES DE LA CIUDAD DE ARMENIA, COLOMBIA

ORAL HEALTH STATUS ON PREGNANT WOMEN IN ARMENIA CITY, COLOMBIA

Herney A. Rengifo, Francy Ciro, Alejandra Obando, Lina Roldán, Andrea López, Lina López

13 PREVALENCIA DE CARIES Y ALTERACIONES DENTARIAS EN NIÑOS CON LABIO FISURADO Y PALADAR HENDIDO DE UNA FUNDACIÓN DE SANTIAGO DE CALI

CARIES AND DENTAL ALTERATION PREVALENCE IN CLEFT LIP AND PALATE CHILDREN IN A FUNDATION OF SANTIAGO DE CALI

Ana M. Hurtado, Lina M. Rojas, Diana M. Sánchez, Ana M. García, María A. Ortíz, Andrés F. Aguirre

18 EFECTOS DEL PERÓXIDO DE HIDRÓGENO ACTIVADO CON LUZ ULTRAVIOLETA Y EL PERÓXIDO DE CARBAMIDA EN ACLARAMIENTO DENTAL

EFFECT OF HYDROGEN PEROXIDE ACTIVATED BY ULTRAVIOLET LIGHT AND CARBAMIDE PEROXIDE IN TEETH BLEACHING

Adriana Cabrera, Melissa David, luisa S. Pacheco, Ana C. Suárez, Herney Garzón

Revisiones de Tema

25 REABSORCIÓN RADICULAR INDUCIDA POR ORTODONCIA

ROOT REABSORTION INDUCE BY ORTHODONTIC PROCEDURES

Angie Clarkson

30 DIFERENCIAS ENTRE EL NIVEL DE SIGNIFICANCIA ALFA Y EL VALOR P

DIFFERENCES BETWEEN ALFA SIGNIFICANCE LEVEL AND P VALUE

HÉCtOR F. Mueses 


\section{Revista Estomatología}

\section{Contribuciones}

La Revista Estomatología tiene especial interés en publicar colaboraciones originales inéditas, que abarquen las ciencias naturales y humanas, la biotecnología y la cultura, incluídas las bellas artes, sin que tales intereses alteren la amplitud con que se ofrece esta tribuna a la gente pensante de Colombia y el mundo, pero de manera especial a los estudiantes de los niveles "Auxiliar", "Técnico Simple", "Técnico Profesional", "Tecnológico" y "Profesional" de pregrado y postgrado, a los profesores de la Escuela, a los de las otras Escuelas de la Facultad de Salud incluida la Escuela de Salud Pública y sus centros especiales (CEDETES, CISALVA, etc.) así como a estudiantes y profesores del Instituto de Psicología, de la Facultad de Comuncación Social y, en general, a los investigadores en odontoestomatología.

La Revista Estomatología pretende continuar como una tribuna de divulgación científica, dedicada a difundir con un estiloágil y atractivo, así como con enfoques adecuados al lector no especializado, conceptos, ideas, métodos y resultados de investigaciones. También avances sobre estudios en ejecución, sin detallar a profundidad el desarrollo de investigaciones en curso.

La Revista Estomatología incorpora contribuciones de naturaleza distinta así:

Editoriales. Documentos que reflejan la posición del Consejo Editorial de la Revista frente a un determinado problema o tópico. Ordinariamente son producidos por el Director o por el Editor Asociado de la Revista Estomatología pero, también pueden ser elaborados por personas diferentes a los miembros del Consejo, a petición expresa del mismo. Para esta sección no se aceptan contribuciones espontáneas.

Cartas al Editor. Comunicaciones breves que busquen aclarar o complementar conceptos originados en los contenidos de los artículos publicados en la revista Estomatología.

Trabajos de Investigación y Artículos Originales. Incluye los informes finales de los trabajos de indagación científica que pueden ser de interés para los lectores de la Revista. Las contribuciones para esta sección se acogen a las normas que rigen la literatura odontológica científica mundial. Los detalles aparecen en las Normas de Publicación.
Literatura Odontológica. Se aceptan contribuciones de excepcional interés que hayan sido publicadas en otras revistas del exterior. En este caso, siempre se deberá mencionar si se ha publicado previamente y se deberán incluir la referencia original y el permiso del correspondiente editor.

Actualización en Salud Oral. Se pueden presentar revisiones completas y exhaustivas sobre diferentes tópicos, cuyo propósito sea poner al día a los lectores respecto a los avances en el campo de la ciencia. Los contribuyentes serán personas de reconocido prestigio en el campo de su práctica y, ordinariamente, deben incluir abundantes referencias recientes.

Comunicaciones Breves. Notas cortas, máximo dos páginas tamaño carta escritas a doble espacio, en las cuales se trata de expresar una enseñanza sobre un tema específico o comentar un problema reciente o plantear una inquietud. Su bibliografía se limita a 10 referencias.

Contribuciones de Artes y Cultura. Se aceptan artículos de interés en relación con la literatura (narrativa, poesía, ensayos filosóficos) o con las manifestaciones de las artes plásticas. La condición es que tengan los requerimientos de las comunicaciones breves.

Los artículos publicados en la Revista Estomatología son de responsabilidad exclusiva del Autor (o Autores) y no reflejan necesariamente el criterio o el pensamiento del Consejo Editorial.

Dirigir todos los manuscritos y correspondencia a:

\section{Revista Estomatología}

odontologia@univalle.edu.co

Dr. José Fernando Barreto, Editor Asociado

Escuela de Odontología, Facultad de Salud

Universidad del Valle, Sede San Fernando

Calle $3 A N^{\circ} 36 B-00$, Edificio 132, Oficina 307

Apartado Aéreo 25360

Cali, Colombia 


\section{Editorial}

$\mathrm{D}$ urante los últimos años los esfuerzos académicos realizados por las instituciones educativas locales en el desarrollo y creación de nuevo conocimiento en Odontología se ha multiplicado. Es evidente que un buen número de estos temas de investigación no presentan un enfoque científico dirigido a responder las necesidades de los problemas de salud oral de los Colombianos y al contrario se limitan a problemas puntuales, en muchas ocasiones sin relevancia, pero suficientes para el logro de requisitos de grado en algunos programas de pregrados y de especializaciones.

La dificultad para realización de investigaciones en Odontología que presenten niveles de evidencia altos (Ensayos clínicos, Estudios analíticos) podría deberse a diferentes factores como son: la imposibilidad de diseñar estudios de seguimiento en grupos que podrian tardar años en presentar reacciones adversas o fracasos en los tratamientos y a los altos costos que generaría el tratamiento de una muestra capaz de mostrar resultados de significancia estadística. En general, y no sólo en nuestro país el Odontólogo es formado con bases investigativas débiles, lo cual genera una cierta desestimación por el manejo sistemático de los registros clínicos, entorpeciendo asi la realización de estudios retrospectivos de calidad; esto, unido a la poca concentración de odontólogos expertos en investigación (muchas veces provocado por la poca motivación que presenta el sistema para este tipo de especializaciones en odontología) que apoyen estos trabajos con el fin de fortalecerlos tanto teórica como metodológicamente.

Muchas de las instituciones que han querido entrar en el mundo de la investigación, concientes de la importancia que esto representa para su acreditación tanto nacional como internacional, lo hacen con grandes limitaciones académicas y presupuestales, lo cual restringe el logro de estudios veraces; sin embargo, este gran esfuerzo debe ser reconocido; en un entorno en cual las cátedras de investigación tienden a ser desestimadas al enfrentarlas a las actividades clínicas propias de un sistema operativo y poco preventivo en salud.

El desarrollo de la investigación no radica en el aumento en la cantidad de los estudios, sino en la calidad que estos pudiesen presentar y su relevancia para la comunidad científica nacional e internacional.

En un país en vía de desarrollo como el nuestro, es necesario unir esfuerzos en procura de un aumento en la calidad de nuestras investigaciones. Es evidente la fortaleza de algunas universidades en ciertos temas específicos, las cuales podrían apoyar a otras en el desarrollo de sus trabajos, mediante la realización de pactos de colaboración nacional, para generar nuevas fuentes de ocupación e intercambios interinstitucionales para los profesionales motivados por los procesos académicos.

No podemos dejar a un lado la necesidad de fortalecer las revistas existentes para lograr un medio de divulgación de calidad en la cual nuestros investigadores puedan publicar y ser referenciados por la comunidad científica internacional.

Andrés Felipe Aguirre $O$. Editor Invitado. Santiago de Cali, Julio 2008 